\title{
Long-Range Interaction and Synchronization of Oscillating Dissipative Solitons
}

\author{
D. Turaev, ${ }^{1}$ A. G. Vladimirov, ${ }^{2}$ and S. Zelik ${ }^{3}$ \\ ${ }^{1}$ Imperial College, London SW7 2AZ, United Kingdom \\ ${ }^{2}$ Weierstrass Institute, Mohrenstrasse 39, D-10117 Berlin, Germany \\ ${ }^{3}$ University of Surrey, Guildford, GU2 7XH, United Kingdom \\ (Received 8 November 2011; published 28 June 2012)
}

\begin{abstract}
We study the interaction of well-separated oscillating localized structures (oscillons). We show that oscillons emit weakly decaying dispersive waves, which lead to the formation of bound states due to harmonic synchronization. We also show that in optical applications the Andronov-Hopf bifurcation of stationary localized structures leads to a drastic increase in their interaction strength.
\end{abstract}

DOI: 10.1103/PhysRevLett.108.263906

PACS numbers: 42.65.Sf, 47.54. $-\mathrm{r}$

The investigation of localized structures arising in physical systems of various nature is an important subject of nonlinear science. Lately a lot of attention has been paid to the so-called dissipative solitons [1]. Their formation requires a balance of energy gain and dissipation, which makes the dissipative solitons more stable to perturbations and, therefore, more attractive for practical applications (e.g., for optical information processing) than the classical solitons of integrable Hamiltonian equations. Exact analytical expressions for dissipative solitons are rarely available, so qualitative methods become especially important in their study. An interesting problem which can be treated by qualitative methods is the interaction of dissipative solitons [2-8]. While most of the studies here were focused on the case of stationary solitons, in this Letter we analyze the interaction of dissipative solitons which oscillate in time.

It is well known that a stationary soliton can exhibit instabilities that lead to various dynamical regimes. One of the simplest and most frequently encountered between these instabilities is the Andronov-Hopf (AH) bifurcation resulting in undamped pulsations of the soliton's parameters, such as amplitude, width, etc. [1,8-10]. Here, we show that the transition from stationary to an oscillating soliton (oscillon) leads to the formation of various new types of multisoliton bound states. In particular, the AH bifurcation of stationary optical pulses results in a considerable increase of their interaction strength.

Although the approach we use is general, to illustrate the enhancement of the solitons' interaction, we consider a specific model equation (Lugiato-Lefever model [11])

$$
\partial_{t} a=(i+\epsilon) \partial_{x x} a-(\gamma+i \theta) a+i a|a|^{2}+2 p,
$$

which describes formation of transverse patterns in Kerr cavity [10] or "temporal cavity solitons" in fibers [12]. Here, $a$ is the field envelope, $\gamma$ is the cavity decay rate, $\theta$ is the cavity detuning, and $p$ is the external coherent pumping. Spatial filtering (or, in time domain, gain dispersion) $\epsilon$ is typically quite small in optical applications.
The soliton in Eq. (1) is asymptotic to a nonzero stationary value $a_{s}(p)$. The existence region of the soliton is presented in the bifurcation diagram in Fig. 1. As the pumping parameter $p$ increases above the critical value $p_{A H}$, the soliton undergoes an AH bifurcation [10]. After this bifurcation, the soliton starts to oscillate, and the oscillations in the tail can be interpreted as a radiation of evanescent waves, see Fig. 2 for an illustration.

In order to find the dispersion relation which determines the decay rate of these waves, we perform linear stability analysis near the stationary homogeneous solution. We let $a-a_{s}=e^{\lambda x}\left(b e^{i \omega t}+c e^{-i \omega t}\right)$ in Eq. (1) linearized about the homogeneous background. This gives a biquadratic equation:

$$
\begin{aligned}
& \left\{i \omega-\lambda^{2}(i+\epsilon)+\left[\gamma+i\left(\theta-2\left|a_{s}\right|^{2}\right)\right]\right\} \\
& \quad \times\left\{i \omega+\lambda^{2}(i-\epsilon)+\left[\gamma-i\left(\theta-2\left|a_{s}\right|^{2}\right)\right]\right\}=\left|a_{s}\right|^{4} .
\end{aligned}
$$

The two solution branches with $\operatorname{Re} \lambda<0$ determine the spatial decay rates corresponding to the oscillations in time with the frequency $\omega$ [these two branches are related by
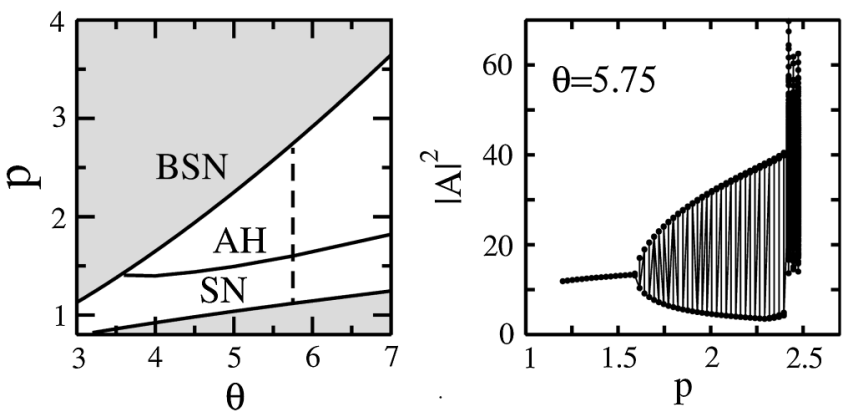

FIG. 1. Left: bifurcation diagram for Eq. (1) at $\epsilon=0.02$, $\gamma=1$. BSN-saddle-node bifurcation of the spatially homogeneous solution. SN-saddle-node bifurcation of the stationary soliton. AH-Andronov-Hopf bifurcation of the soliton. Stable soliton is born at the curve $\mathrm{SN}$, and it becomes an oscillon above $\mathrm{AH}$. Above the line BSN, the homogeneous background disappears and no solitons can exist. Right: soliton "bifurcation tree" for $\theta=5.75$ (dashed line in the left panel). 

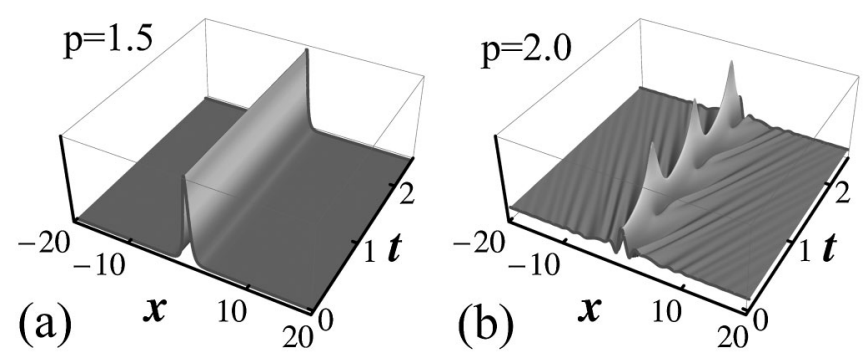

FIG. 2. Stable stationary (a) and oscillating (b) solitons of Eq. (1), numerically obtained by the method of lines with 5-point approximation of the Laplacian (periodic boundary conditions on the uniform grid of 512 nodes in the spatial interval $x \in[-35,35])$ and implicit Gear's integrator [14]. In all the figures, here and below, $\epsilon=0.02, \gamma=1, \theta=5.75$.

$\left.\lambda_{1}(\omega)=\lambda_{2}^{*}(-\omega)\right]$. At $\epsilon=0$, we have $\operatorname{Re} \lambda_{1}(\omega) \rightarrow 0$ as $\omega \rightarrow+\infty$. Therefore, at small $\epsilon$, the maximal value of $\operatorname{Re} \lambda$ is achieved at large $\omega$ and is close to zero, moreover the maximum is flat (see Fig. 3). Thus, for a large range of sufficiently high frequencies the dispersive waves are damped only weakly and can propagate to sufficiently large distances. This is the key mechanism behind the long-range synchronization of oscillons reported below.

Figure 4 shows the numerically calculated distance between two solitons in Eq. (1), as a function of time. Below the bifurcation threshold $p=p_{A H}$, the distance stays constant; i.e., the exponentially decaying interaction strength between stationary solitons is negligibly small for chosen intersoliton distances [Fig. 4(a)]. This is in agreement with the experimental findings of Ref. [12] where, for solitons in a coherently driven fiber cavity, the effective stop of the interaction is reported as the intersoliton distance exceeds a certain threshold (the saddle steady state of the soliton interaction equation, in our interpretation). Above the onset of self-oscillations, the picture changes drastically [Fig. 4(b)]: the oscillons visibly move and form numerous bound states distinguished by the intersoliton distance and the difference in the oscillation phases.

We explain this by the fact that the strength of interaction between two well-separated solitons is determined mostly by the rate of soliton's tail decay. For stationary solitons, this rate is given by a single exponent that dominates the tail. In contrast to that, for the solitons oscillating in time at the frequency $\Omega$, each harmonic frequency $n \Omega$ determines its own spatial decay rate, which can be much slower than that of the stationary component. Indeed, the oscillon tails should be viewed as formed out of the dispersive waves corresponding to $\omega=n \Omega$. This gives us the following asymptotic behavior for the tail:

$$
a-a_{s} \sim \sum_{n} b_{n} e^{\lambda(n \Omega) x+i n \Omega t}+c_{n} e^{\lambda^{*}(n \Omega) x-i n \Omega t},
$$

where $x$ is the distance to the soliton center, and $\lambda(\omega)$ are given by (2). The balance between the spatial decay rates $\lambda_{n}$ and the coefficients $b_{n}, c_{n}$ determines which terms
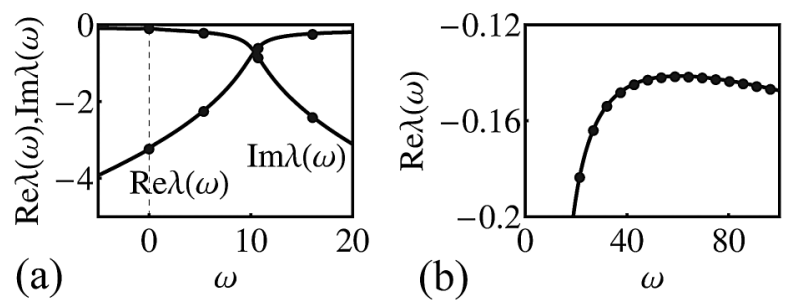

FIG. 3. Dispersion relation at $p=2$ (the main features of the dispersion relation hold for a wide parameters' range, e.g., we obtain a similar graph at $a_{s}=0$ ). (a) Real and imaginary parts of $\lambda(\omega)$ obtained by solving Eq. (2). (b) The maximum of the real part of $\lambda$ is very flat. Black dots correspond to $\omega=n \Omega$, where $\Omega \approx 5.2$ is the oscillon main frequency.

dominate expansion (3) at a given range of $x$. In principle, the slowest decaying exponent corresponds to the maximum of $\operatorname{Re} \lambda$. However, at small $\epsilon$ this maximum is achieved at large $n$ (see Fig. 3), which corresponds to small $b_{n}, c_{n}$ (being Fourier coefficients of an analytic function, they fall exponentially with $n$ ). Therefore, the decay rate of the oscillon tail can slow down with the increase of $x$. Moreover, several exponents can give comparable contribution to the tail at certain spatial scales due to the flatness of the maximum of the dispersion relation. In Fig. 5, we see that the two main exponents correspond to the frequencies $n \Omega$ with $n=1$ and $n=2$. The stationary component $(n=0)$ decays much faster; hence, the oscillon's interaction by exchanging weakly decaying traveling waves acquires a much longer range than the interaction of stationary solitons.

In order to understand details of the oscillon interaction, let us derive the oscillon interaction equations. By plugging $a=a_{s}+A$ into Eq. (1), we can write it as

$$
\partial_{t} \mathbf{A}=\mathcal{L} \mathbf{A}+\mathbf{f}(\mathbf{A})
$$

where $\mathcal{L}$ is a linear differential operator with constant coefficients, $\mathbf{f}(0)=0, \mathbf{f}^{\prime}(0)=0$, and $\mathbf{A}=(\operatorname{Re} A, \operatorname{Im} A)^{T}$.
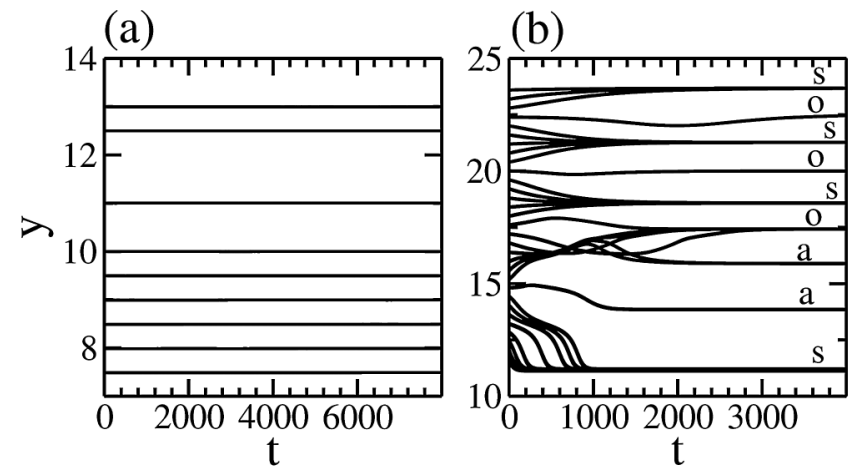

FIG. 4. The intersoliton distance $y(t)$. Different lines correspond to different initial soliton separations and phase differences. (a) $p=1.5$ : two stationary solitons below the $\mathrm{AH}$ threshold. (b) $p=2$ : two oscillons; solutions converge to inphase oscillating bound states, $s$, antiphase states, $a$, and states with the oscillation phase difference $\approx \pi / 2, o$. 

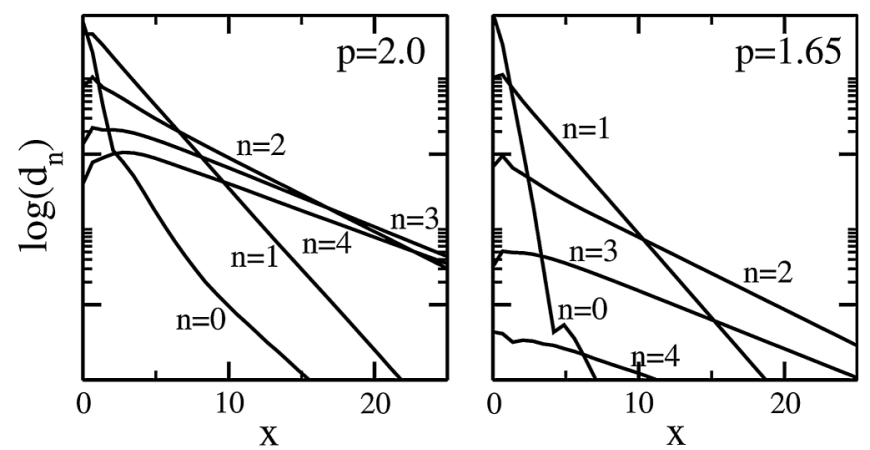

FIG. 5. Absolute value $d_{n}$ of the Fourier coefficients of the oscillon at the frequency $n \Omega$ as functions of the distance $x$ to the soliton center. As $x$ grows, more harmonics contribute to the oscillon tail, while the zero harmonics remains suppressed.

Let $\mathbf{A}_{0}(x, t)=\mathbf{A}_{0}(-x, t)$ be a symmetric oscillon solution of Eq. (1), so $\mathbf{A}_{0}(x, t) \rightarrow 0$ as $x \rightarrow \pm \infty$ and $\mathbf{A}_{0}(x, t)=$ $\mathbf{A}_{0}(x, t+T)$ where $T=2 \pi / \Omega$. Since Eq. (4) is invariant with respect to space and time shifts, the neutral mode equation $-\partial_{t} \boldsymbol{\psi}+\mathcal{L} \boldsymbol{\psi}+\mathbf{f}^{\prime}\left(A_{0}\right) \boldsymbol{\psi}=0$ has two natural solutions $\boldsymbol{\xi}=\partial_{x} \boldsymbol{A}_{0}$ and $\boldsymbol{\eta}=\partial_{t} \boldsymbol{A}_{0}$. We assume there are no other critical modes; i.e., we are sufficiently above the AH instability threshold. The adjoint neutral modes $\boldsymbol{\psi}=$ $\boldsymbol{\xi}^{\dagger}$ (odd in $x$ ) and $\boldsymbol{\psi}=\boldsymbol{\eta}^{\dagger}$ (even in $x$ ) satisfy $\partial_{t} \boldsymbol{\psi}+$ $\mathcal{L}^{\dagger} \boldsymbol{\psi}+\left[\mathbf{f}^{\prime}\left(A_{0}\right)\right]^{\dagger} \boldsymbol{\psi}=0$ with the normalization condition $\int_{0}^{T} d t \int_{-\infty}^{+\infty} d x \boldsymbol{\xi}^{\dagger} \cdot \boldsymbol{\xi}=\int_{0}^{T} d t \int_{-\infty}^{+\infty} d x \boldsymbol{\eta}^{\dagger} \cdot \boldsymbol{\eta}=T$.

We look for the solution of Eq. (4) in the form of two interacting oscillons plus a small correction:

$$
\mathbf{A}=\mathbf{A}_{1}(x, t)+\mathbf{A}_{2}(x, t)+\boldsymbol{\chi}(x, t),
$$

where $\boldsymbol{\chi}$ is small, $\mathbf{A}_{1,2}=\mathbf{A}_{0}\left(x-y_{1,2}, t-\tau_{1,2} / \Omega\right)$, and $y_{1,2}$ and $\tau_{1,2}$, the coordinates and the oscillation phases of the solitons, are slowly varying functions of time. By performing asymptotic expansions, similar to what is done for stationary solitons $[4-7,13]$, we obtain the leading order approximation for the oscillon interaction equation:

$$
\begin{aligned}
T \frac{d y_{1,2}}{d t} & =\int_{0}^{T} \int_{-\infty}^{+\infty} \boldsymbol{\xi}_{1,2}^{\dagger} \cdot \mathbf{S} d x d t, \\
2 \pi \frac{d \tau_{1,2}}{d t} & =\int_{0}^{T} \int_{-\infty}^{+\infty} \boldsymbol{\eta}_{1,2}^{\dagger} \cdot \mathbf{S} d x d t,
\end{aligned}
$$

where $\boldsymbol{\psi}_{1,2}=\boldsymbol{\psi}\left(x-y_{1,2}, t-\tau_{1,2} / \Omega\right)$ (here $\boldsymbol{\psi}=\boldsymbol{\xi}^{\dagger}$ or $\left.\boldsymbol{\psi}=\boldsymbol{\eta}^{\dagger}\right)$ and $\mathbf{S}=\mathbf{f}\left(\mathbf{A}_{1}+\mathbf{A}_{2}\right)-\mathbf{f}\left(\mathbf{A}_{1}\right)-\mathbf{f}\left(\mathbf{A}_{2}\right)$. We assume the oscillons are well separated; i.e., $y_{2}-y_{1}$ is large, so the overlap function $\mathbf{S}$ is small. The oscillon tails decay fast, so $\mathbf{S} \approx \mathcal{M}_{1} \mathbf{A}_{2}$ at $x<y_{*}$ and $\mathbf{S} \approx \mathcal{M}_{2} \mathbf{A}_{1}$ at $x>y_{*}$, where $\mathcal{M}_{1,2}=\mathcal{L}-\partial_{t}+\mathbf{f}^{\prime}\left(\mathbf{A}_{1,2}\right)$, and $y_{*}=\left(y_{1}+y_{2}\right) / 2$ is the middle point of the two-oscillon configuration. Since $\boldsymbol{\xi}_{j}^{\dagger}$ and $\boldsymbol{\eta}_{j}^{\dagger}$ are localized near $x=y_{j}$, we have

$$
\begin{aligned}
T \frac{d y_{j}}{d t} & \approx \int_{0}^{T} \int_{I_{j}} \boldsymbol{\xi}_{j}^{\dagger} \cdot \mathcal{M}_{j} \mathbf{A}_{k} d x d t, \\
2 \pi \frac{d \tau_{j}}{d t} & \approx \int_{0}^{T} \int_{I_{j}} \boldsymbol{\eta}_{j}^{\dagger} \cdot \mathcal{M}_{j} \mathbf{A}_{k} d x d t,
\end{aligned}
$$

where $k=3-j$, and $I_{1}=\left[-\infty, y_{*}\right], I_{2}=\left[y_{*},+\infty\right]$. Now, using the relations $\mathcal{M}_{j}^{\dagger} \boldsymbol{\xi}_{j}^{\dagger}=0, \mathcal{M}_{j}^{\dagger} \boldsymbol{\eta}_{j}^{\dagger}=0$, one takes the integrals with respect to $x$. In our case, where the operator $\mathcal{L}$ is defined by Eq. (1), we finally obtain

$$
\begin{aligned}
T \frac{d y_{j}}{d t} & =(-1)^{j} \int_{0}^{T}\left(\partial_{x} \boldsymbol{\xi}_{j}^{\dagger} E \mathbf{A}_{k}-\boldsymbol{\xi}_{j}^{\dagger} E \partial_{x} \mathbf{A}_{k}\right)_{x=y_{*}} d t, \\
2 \pi \frac{d \tau_{j}}{d t} & =(-1)^{j} \int_{0}^{T}\left(\partial_{x} \boldsymbol{\eta}_{j}^{\dagger} E \mathbf{A}_{k}-\boldsymbol{\eta}_{j}^{\dagger} E \partial_{x} \mathbf{A}_{k}\right)_{x=y_{*}} d t,
\end{aligned}
$$

where $E=\left(\begin{array}{cc}\epsilon & -1 \\ 1 & \epsilon\end{array}\right)$. As we see, the evolution of the interacting oscillons is, to the first order, determined by the asymptotics of their tails and of the adjoint neutral modes $\boldsymbol{\xi}^{\dagger}$ and $\boldsymbol{\eta}^{\dagger}$ and does not depend on the specific form of the nonlinearity $\mathbf{f}$. Plugging the asymptotic formula (3) in the obtained interaction equations, we find

$\frac{d y}{d t}=\sum_{n=-\infty}^{\infty} B_{n} e^{-\alpha_{n} y} \sin \left(\beta_{n} y+\Theta_{1 n}\right) \cos (n \tau)$,

$\frac{d \tau}{d t}=\sum_{n=-\infty}^{\infty} C_{n} e^{-\alpha_{n} y} \cos \left(\beta_{n} y+\Theta_{2 n}\right) \sin (n \tau)$,

where $\alpha_{n}=-\operatorname{Re} \lambda(n \Omega), \beta_{n}=\operatorname{Im} \lambda(n \Omega), y=y_{2}-y_{1}$, $\tau=\tau_{2}-\tau_{1}$, and the coefficients $B, C, \theta$ are expressed via the Fourier coefficients $b_{n}$ and $c_{n}$ and analogous coefficients of the asymptotic expansions for $\boldsymbol{\xi}^{\dagger}$ and $\boldsymbol{\eta}^{\dagger}$.

The main contribution to the sums in Eq. (5) is typically made by a small number of exponents which correspond to the minimal values of $\alpha_{n}$ and, at moderate $y$, to the maximal values of $B_{n}, C_{n}$. Consider the case where only one term dominates in the sum. If it corresponds to the zero harmonics $n=0$ (i.e. $\alpha_{0}<\min _{n \neq 0} \alpha_{n}$ ), then the $y$-equation does not, to the leading order, depend on $\tau$. Then, the distance between the oscillons behaves like in the stationary case [5-7,13]: at $\beta_{0} \neq 0$ stable bound states are formed near $\beta_{0} y+\theta_{10}=\pi(2 k+1)$, independently of the value of the phase difference $\tau$. Possible phase synchronization effects appear on a much longer time scale and are governed by nonzero harmonics.

If the dominating exponent corresponds to a nonzero harmonic $N \neq 0$, then the oscillon interaction equations reduce to

$$
\begin{aligned}
& \frac{d y}{d t}=B e^{-\alpha_{N} y} \sin \left(\beta_{N} y+\theta_{1 N}\right) \cos (N \tau), \\
& \frac{d \tau}{d t}=C e^{-\alpha_{N} y} \cos \left(\beta_{N} y+\theta_{2 N}\right) \sin (N \tau) .
\end{aligned}
$$

When $N=1$, Eqs. (6) coincide with those derived in [4,7] for the interaction of stationary solitons in complex Ginzburg-Landau (CGL) type models (unlike Eq. (1), CGL equations have a phase-shift symmetry $a \rightarrow a e^{i \phi}$, so $\tau=\phi_{2}-\phi_{1}$ describes the difference between the corresponding phases of the stationary solitons). By borrowing the results of the analysis of the stationary solitons' interaction in CGL [3,4,7,8], we find that Eq. (6) at $N=1$ has three different sets of steady states synchronized with the phase differences $\tau=0, \pi, \pm \pi / 2$. Depending on the 


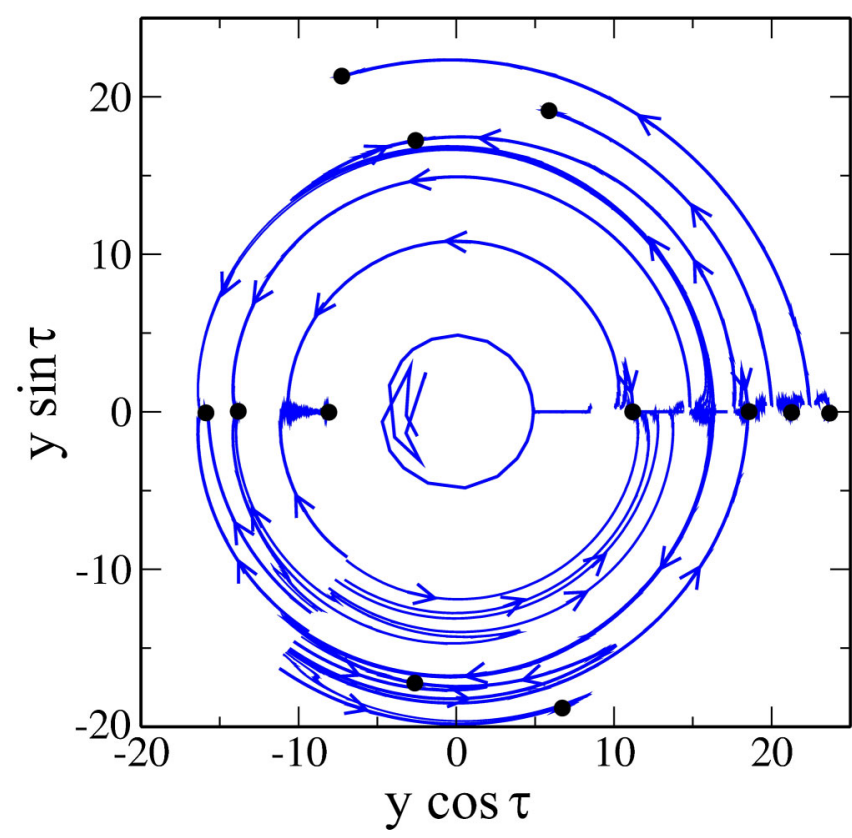

FIG. 6 (color online). Poincaré map for the evolution of two interacting oscillons $(p=2.0)$. For various initial conditions, consecutive values of the intersoliton distance $y$ and the phase difference $\tau$ are shown at the time moments the amplitude of the left oscillon in the pair takes its maximal value. Large dots indicate stable oscillon bound states. At distances $y>4.8$, the discrete trajectories closely follow continuous lines, as predicted by Eq. (5), while at smaller distances $y$ the theory of weak oscillon interaction is not applicable.

parameter values, Eq. (6) demonstrates two different types of dynamical behavior [4]. If $B C \cos \left(\theta_{21}-\theta_{11}\right)>0$, the only attractors are in-phase and antiphase bound states. On the contrary, for $B C \cos \left(\theta_{21}-\theta_{11}\right)<0$, the in-phase and antiphase bound state are unstable, and solutions of Eq. (6) oscillate around the $\pm \pi / 2$ out-of-phase bound states. In the full system, the in-phase or antiphase oscillon bound states are preserved, while the phase shift for the out-ofphase bound states can slightly differ from $\pi / 2$ [since higher order corrections destroy the reversibility of Eq. (6)]. The phase portrait for the case $N>1$ is formally recovered from that for $N=1$ by rescaling $\tau$. However, a novel phenomenon of the superharmonic oscillon synchronization emerges: stable bound states with the phase differences $\tau \approx \pi k /(2 N)$ become possible.

The results of numerical simulations of two-oscillon interaction in Eq. (1) are presented in Fig. 6. It is seen from this figure that when the oscillon separation is sufficiently small, we have only in-phase and antiphase stable bound states, which is typical for Eq. (6) with $N=1$ and $B C \cos \left(\theta_{2 N}-\theta_{1 N}\right)>0$. However, at larger oscillon separations, stable bound states with the phase difference around $\pi / 2$ appear and the phase portrait becomes consistent with Eq. (6) in the case $N=2$. In particular, the sequence of in-phase bound states becomes equidistant with the increment $\approx 1.3$, close to $\pi / \beta_{2}$.
To explain this, recall that a single exponent is not sufficient for the description of the oscillon tail asymptotics of Eq. (1) for the chosen set of parameters (see Fig. 5). By retaining the two terms corresponding to $n=1$ and $n=2$ in Eqs. (5), we obtain:

$$
\begin{aligned}
\frac{d y}{d t}= & B_{1} e^{-\alpha_{1} y} \sin \left(\beta_{1} y+\Theta_{11}\right) \cos \tau \\
& +B_{2} e^{-\alpha_{2} y} \sin \left(\beta_{2} y+\Theta_{12}\right) \cos 2 \tau, \\
\frac{d \tau}{d t}= & C_{1} e^{-\alpha_{1} y} \cos \left(\beta_{1} y+\Theta_{21}\right) \sin \tau \\
& +C_{2} e^{-\alpha_{2} y} \cos \left(\beta_{2} y+\Theta_{22}\right) \sin 2 \tau .
\end{aligned}
$$

Since $\alpha_{1}>\alpha_{2}$, the terms with $2 \tau$ begin to dominate in this equation with the increase of the oscillon separation $y$. The phase portrait shown in Fig. 6 is consistent with Eq. (7) for $B_{2} / B_{1} \approx C_{2} / C_{1} \approx 0.02$. At even larger distances, numerical simulations reveal stable bound states with $\tau \approx \pm \pi / 3$, $2 \pi / 3$ which should correspond to higher harmonics coming into play.

To conclude, we have shown that the transition from stationary to oscillating solitons can lead to a drastic enhancement of the soliton interaction strength. This is especially true in many optical applications where the diffusion $\epsilon$ is typically small: in this case the highfrequency linear waves emitted by the oscillons have a low dissipation rate and, therefore, are the main agent of the weak interaction (this is also true in two spatial dimensions). Different bound states of oscillons are distinguished by the distance between them and the oscillons' phase difference; i.e., they correspond to different oscillon synchronization regimes. We have found that harmonic synchronization is a typical phenomenon here.

This research was supported by SFB 787 of the DFG, EU FP7 Grant No. 264687, MES of Russia Grant No. 2011-1.5503-002-038, the Leverhulme Trust Grant No. RPG-279, and an E. T.S. Walton Visitor Award of the SFI.

[1] Dissipative Solitons, Lecture Notes in Physics Vol. 661, edited by N. Akhmediev and A. Ankiewicz (Springer, Berlin, 2005); Dissipative Solitons: from Optics to Biology and Medicine, Lecture Notes in Physics Vol. 751 (Springer, Berlin, 2008).

[2] B. A. Malomed, Phys. Rev. A 44, 6954 (1991); K. Gorshkov, A. Lomov, and M. Rabinovich, Nonlinearity 5, 1343 (1992); B. Schäpers, M. Feldmann, T. Ackemann, and W. Lange, Phys. Rev. Lett. 85, 748 (2000); N. N. Rosanov, S. V. Fedorov, and A. N. Shatsev, Phys. Rev. Lett. 95, 053903 (2005); D. Turaev and S. Zelik, Discrete Contin. Dyn. Syst. A 28, 1713 (2010).

[3] V. V. Afanasjev and N. N. Akhmediev, Phys. Rev. E 53, 6471 (1996); N. N. Akhmediev, A. Ankiewicz, and J. M. Soto-Crespo, J. Opt. Soc. Am. B 15, 515 (1998); N. Akhmediev, J. M. Soto-Crespo, M. Grapinet, and $\mathrm{Ph}$. Grelu, Opt. Fiber Technol. 11, 209 (2005). 
[4] A. G. Vladimirov, G. V. Khodova, and N. N. Rosanov, Phys. Rev. E 63, 056607 (2001).

[5] A. G. Vladimirov, J. M. McSloy, D. V. Skryabin, and W. J. Firth, Phys. Rev. E 65, 046606 (2002).

[6] B. Sandstede, in Handbook of Dynamical Systems (NorthHolland, Amsterdam, 2002), Vol. II, pp. 983-1055.

[7] S. Zelik and A. Mielke, Mem. Am. Math. Soc. 198, 1 (2009).

[8] D. Turaev, A. G. Vladimirov, and S. Zelik, Phys. Rev. E 75, 045601(R) (2007).

[9] B.S. Kerner and V.V. Osipov, Autosolitons. A New Approach to Problems of Self-Organization and Turbulence, Fundamental Theories of Physics Vol. 61 (Springer, 1994); D. Haim, G. Li, Q. Ouyang, W. D. McCormick, H. L. Swinney, A. Hagberg, and E. Meron, Phys. Rev. Lett. 77, 190 (1996); A. G. Vladimirov, N. N. Rosanov, S. V. Fedorov, and G. V. Khodova, Quantum Electron. 28, 55 (1998); D. Michaelis, U. Peschel, and F. Lederer, Opt. Lett. 23, 1814 (1998); A. G. Vladimirov, S. V. Fedorov, N. A. Kaliteevskii, G. V. Khodova, and
N. N. Rosanov, J. Opt. B 1, 101 (1999); N. N. Rozanov, S. V. Fedorov, and A. N. Shatsev, Opt. Spectrosc. 91, 232 (2001); S. V. Gurevich, S. Amiranashvili, and H.-G. Purwins, Phys. Rev. E 74, 066201 (2006).

[10] W. J. Firth, A. Lord, and A. J. Scroggie, Phys. Scr. T67, 12 (1996); W. J. Firth, G. K. Harkness, A. Lord, J. M. McSloy, D. Gomila, and P. Colet, J. Opt. Soc. Am. B 19, 747 (2002); D. Gomila, P. Colet, M. A. Matías, M. San Miguel, and G.-L. Oppo, Proc. SPIE Int. Soc. Opt. Eng. 5975, 59750U (2006).

[11] L. A. Lugiato and R. Lefever, Phys. Rev. Lett. 58, 2209 (1987).

[12] F. Leo, S. Coen, P. Kockaert, S.-P. Gorza, P. Emplit, and M. Haelterman, Nature Photon. 4, 471 (2010).

[13] K. Gorshkov and L. Ostrovsky, Physica (Amsterdam) 3D, 428 (1981).

[14] C. W. Gear, Numerical Initial Value Problems in Ordinary Differential Equations (Prentice Hall, Englewood Cliffs, 1971). 